\title{
Soft Interchangeability for Case Adaptation
}

\author{
Nicoleta Neagu and Boi Faltings \\ Artificial Intelligence Laboratory (LIA), \\ Computer Science Department, Swiss Federal Institute of Technology (EPFL) \\ $\mathrm{CH}-1015$ Ecublens, Switzerland \\ \{Nicoleta.Neagu, Boi.Faltings\}@epfl.ch http://liawww.epfl.ch/
}

\begin{abstract}
In [1] we propose interchangeability based algorithms as methods for solving the case adaptation for the domain of problems which can be expressed as Constraint Satisfaction Problems. In this paper we extend the domain to Soft Constraint Satisfaction Problems and give generic adaptation methods based on soft interchangeability concepts.

Many real-life problems require the use of preferences. This need motivates for the use of soft constrains which allows the use of preferences.

We have defined interchangeability for soft CSPs in [2] by introducing two notions: $(\delta / \alpha)$ substitutability/interchangeability and their algorithms. This paper presents how to build generic adaptation methods based on soft interchangeability. It gives an example of an application of a sales manager system for a car configuration domain and reports test results regarding number of $(\delta / \alpha)$ interchangeability in random generated problems, thus number of adaptation alternatives.
\end{abstract}

Keywords: case adaptation, hard/soft constraint satisfaction problems, interchangeability, substitutability.

\section{Introduction}

In [1], we proposed a generic framework for case adaptation for the domain of problems which can be represented as Classic Constraint Satisfaction Problems(CSP). The CSP model can be applied to a wide range of problems [3], well known examples are: diagnosis [4], planning [5], scheduling [6], robot control [7] and configuration [8] but there are still many real life problems which cannot be precisely defined by using classical constraints only. Soft constraints allow for preference levels to be associated to either constraints, or variables, or tuples within the constraints. In this paper we propose adaptation methods for the domains of problems where the knowledge can be represented as a Soft Constraint Satisfaction Problem. Certain domains must be represented as hard constraint satisfaction problems in order for the resulting solution to be functional. For example, in the configuration of a product, the components have to match so that the product is functional. However, many other real-life problems are more naturally handled by using soft constraints $[9,10]$. Classical CSPs consist of a number of choices that need to be made (variables), each of which has an associated number of options (the variable domain) and a set of relationships between choices (constraints). A valid solution to a CSP is an assignment of a value to each variable from its domain with 
the total set of assignments respecting all the problem constraints. In soft CSPs, the significance of the constraints is not that a tuple of values for some variables is allowed or not, but rather the preference level at which it is allowed. One of the frameworks which formalises soft CSPs is the one based on the semiring, see [10]: where each tuple of values in each constraints has an assigned semiring value, to be interpreted as the level of preference of that tuple, or its cost, or any other measurable feature. Further, the constraints are combined according to the semiring operations and the result of the combination is that the assignment for all the variables has a corresponding semiring value too.

In [11], we defined two notions: threshold $\alpha$ and degradation $\delta$ for substitutability and interchangeability, $((\delta / \alpha)$ substitutability/interchangeability). Fortunately, soft constraints also allow weaker forms of interchangeability where exchanging values may result in a degradation of solution quality by some measure $\delta$. By allowing more degradation, it is possible to increase the amount of interchangeability in a problem to the desired level. ${ }^{\delta}$ substitutability/interchangeability is a concept which ensures this quality.

Ideally, one would like to compute values which are interchangeable over global solutions, full interchangeable, but this is computational expensive. That is way, the search of this values is computed in local forms, called neighbourhood interchangeability. Just as for hard constraints, full interchangeability is hard to compute, but can be approximated by neighbourhood interchangeability which can be computed efficiently and implies full interchangeability. We have defined the same concepts for soft constraints, and prove that neighbourhood implies full $(\delta / \alpha)$ substitutability/interchangeability, see [2].

We propose here to apply these techniques in a CBR system where the knowledge domain is represented as a soft CSP. By allowing a certain degradation delta of a case or searching over a certain threshold alpha one can try to realise adaptation. So, in this paper we show how case adaptation can be realised based on these concepts.

The main contribution of this paper is a generic model for the application of soft interchangeability techniques to a large class of CBR adaptation problems (Section 4). We also present an practical product configuration application (Section 2) and examples of our technique within that domain. We conclude the paper with our first experimental results (Section 5) measuring $(\delta / \alpha)$ interchangeability occurrence and thus, the number of adaptation alternatives.

Additionally, in Section 3 we recall the main definitions of soft interchangeability/substitutability that are of interest to us, related work is provided in Section 6 while conclusions and further work can be found in Section 7.

\section{A Product Configuration CBR System.}

The CSP model can be applied to a very wide range of problems. We present here how the framework works for a configuration problem example. However, the framework works in the same way for all the problems which can be represented as a CSP.

We have applied and tested constraint satisfaction based methods in a case based reasoning system which function as a sales manager. The case base reasoning system 
contains cases which represent past sales and consist of a buyer profile and the chosen product. The configuration of the product is modelled as a constraint satisfaction problem. While admissible configurations can be precisely specified as a constraint satisfaction problem, each customer has particular needs that remain largely unformalized. Case-based reasoning maps properties of the customer to these implicit customer needs and product configuration that would fit these needs. Constraint satisfaction techniques can then be used to adapt the proposed solution by the CBR system to the particular customer requirements and preferences.

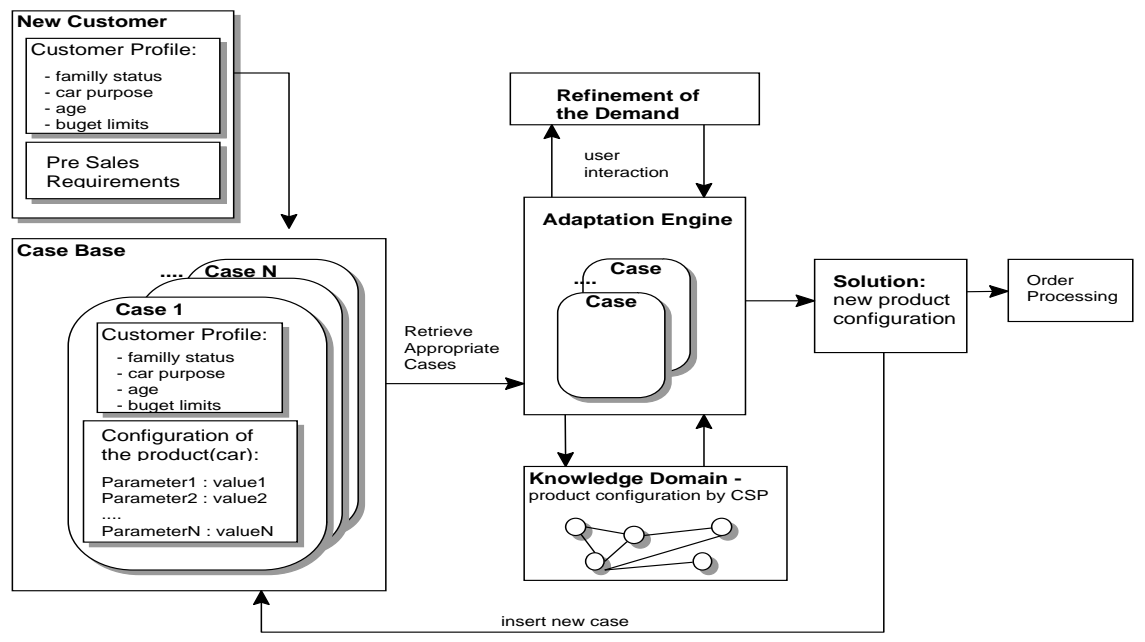

Fig. 1: An electronic sales manager based on a CBR system.

In Figure 1, we represent the general framework of our system. When a new customer makes a new demand, the sales system will search in the case base for similar cases by the use of buyer profile. Each case contains:

- a buyer profile contains:

- characteristics: like age, family status, using purpose for the car, budget limits in the car investment,

- particular preferences/requirements for the car.

- the chosen product which contains the configuration of the car as a constraint satisfaction problem solution where each component of the car has assigned a value consistent with all the other components, represented as variables of the CSP, according to the constraints between them.

The retrieved cases are sent to the adaptation engine which communicates with the buyer for further refinement of the demands; and using interchangeability methods adapts the solutions of the product configuration based on the domain knowledge. The domain knowledge is represented as a constraint satisfaction problem. By interacting with these two modules the adaptation engine reaches the new solution which together 
with the current buyer profile is inserted in the case base. Further, the sale order is send to processing.
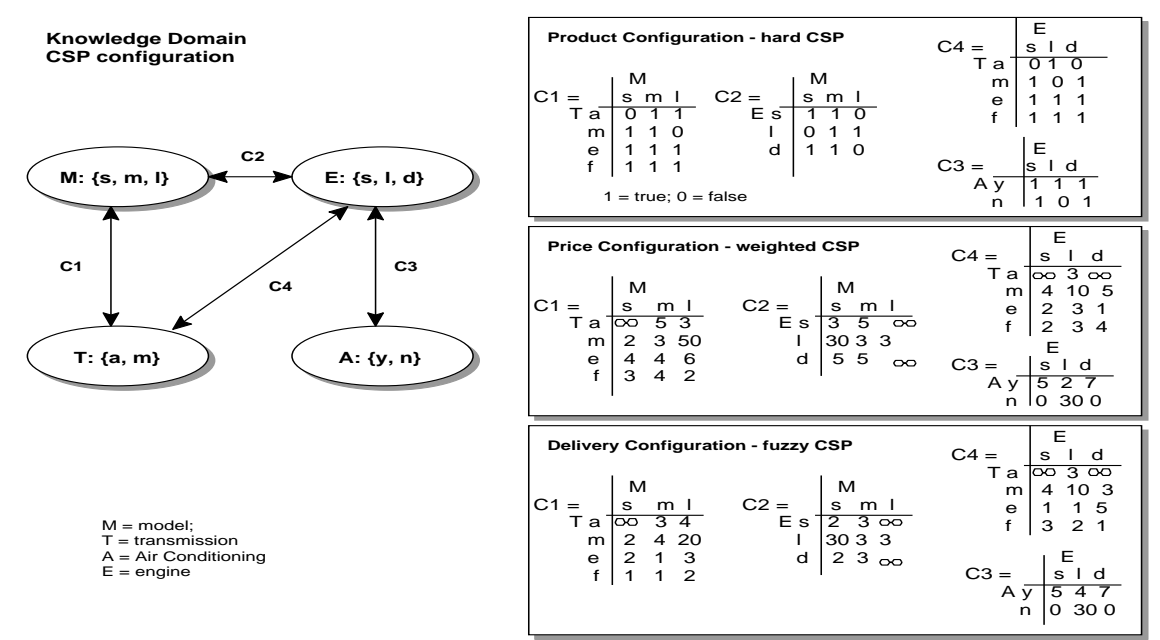

Fig. 2: Domain knowledge as Constraint Satisfaction Problems.

In our example we present three models for representing the knowledge, as shown in Figure 2:

- the first example represents the configuration of the product modelled as a classic (hard) CSP where the constraints are either satisfied or not by a tuple of values; in order that the product is functional all the components have to be compatible with one another.

- in the second example we model the price configuration for the cost of the product as a weighted CSP; the weights on the tuples of values represent the cost for each component (by unary constraints) and the costs for combinations of components respectively (by binary constraints).

- the last example represents the delivery of the product modelled as a fuzzy CSP where the preferences on the tuples of values represent the delivery time of the components and the combinations of components according to the constraints between them.

While the configuration of the product has to be expressed with hard constraints in order that the product is feasible, modelling the price and delivery time with soft constraints allows for the use of customer preferences. The found solutions are then ranked according to the optimisation criteria used by the soft constraint system.

\section{Background}

In the following we give a brief overview of soft CSPs and definitions of different forms of interchangeability. 


\subsection{Soft CSPs}

In many practical applications, constraints can be violated at a cost, and solving a CSP thus means finding a value assignment of minimum cost ${ }^{1}$. Various frameworks for solving such soft constraints have been proposed [12-18]. The soft constraint framework of c-semirings [17] has been shown to express most of the known variants through different instantiations of its operators, and this is the framework we are considering in this paper.

A soft constraint may be seen as a constraint where each instantiation of its variables has an associated value from a partially ordered set which can be interpreted as a set of preference values. Combining constraints will then have to take into account such additional values, and thus the formalism has also to provide suitable operations for combination $(\times)$ and comparison $(+)$ of tuples of values and constraints. This is why this formalization is based on the concept of c-semiring, which is just a set plus two operations, [17].

A $c$-semiring is a semiring(a tuple) $\langle A,+, \times, \mathbf{0}, \mathbf{1}\rangle$ such that: + is commutative, associative, idempotent, $\mathbf{0}$ is its unit element and $\mathbf{1}$ is its absorbing element; $\times$ is associative, distributes over + , commutative, $\mathbf{1}$ is its unit element and $\mathbf{0}$ is its absorbing element.

Soft Constraint Problems. Given a semiring $S=\langle A,+, \times, \mathbf{0}, \mathbf{1}\rangle$ and an ordered set of variables $V$ over a finite domain $D$, a constraint is a function which, given an assignment $\eta: V \rightarrow D$ of the variables, returns a value of the semiring.

By using this notation we define $\mathcal{C}=\eta \rightarrow A$ as the set of all possible constraints that can be built starting from $S, D$ and $V$.

Combining and projecting soft constraints. Given the set $\mathcal{C}$, the combination function $\otimes: \mathcal{C} \times \mathcal{C} \rightarrow \mathcal{C}$ is defined as $\left(c_{1} \otimes c_{2}\right) \eta=c_{1} \eta \times{ }_{S} c_{2} \eta$.

In words, combining two constraints means building a new constraint whose support involves all the variables of the original ones, and which associates with each tuple of domain values for such variables a semiring element which is obtained by multiplying the elements associated by the original constraints to the appropriate subtuples.

Given a constraint $c \in \mathcal{C}$ and a variable $v \in V$, the projection of $c$ over $V-\{v\}$, written $c \Downarrow_{(V-\{v\})}$ is the constraint $c^{\prime}$ s.t. $c^{\prime} \eta=\sum_{d \in D} c \eta[v:=d]$.

Informally, projecting means eliminating some variables from the support. This is done by associating with each tuple over the remaining variables a semiring element which is the sum of the elements associated by the original constraint to all the extensions of this tuple over the eliminated variables. In short, combination is performed via the multiplicative operation of the semiring, and projection via the additive one.

\subsection{Degradations and Thresholds in Soft CSPs}

Interchangeability in constraint networks proposed by Freuder in [?], captures equivalence among the variables values in a discrete CSP. Two values $a$ and $b$ of a CSP

\footnotetext{
${ }^{1}$ Through the paper we measure the quality of solution by the cost of the CSP, where the quality is good when the cost is low.
} 
variable $v$ are interchangeable if for any solution where $v=a$, there is an identical solution except that $v=b$, and vice versa.

The most straightforward generalization of interchangeability to soft CSP would require that exchanging one value for another does not change the quality of the solution at all. This generalization is likely to suffer from the same weaknesses as interchangeability in hard CSP, namely that it is very rare.

Fortunately, soft constraints also allow weaker forms of interchangeability where exchanging values may result in a degradation of solution quality by some measure $\delta$. By allowing more degradation, it is possible to increase the amount of interchangeability in a problem to the desired level. We define ${ }^{\delta}$ substitutability and ${ }^{\delta}$ interchangeability as a concept which ensures this quality. This is particularly useful when interchangeability is used for solution adaptation.

In a hard CSP, a CSP solution is a value assignment to the variables such that all the constraints are satisfied. In soft CSPs, any value assignment is a solution which have a corresponding semiring value. The semiring values represents the preference value of that solution. This allows broadening the original interchangeability concept to one that also allows degrading the solution quality when values are exchanged. We call this $\delta$ interchangeability, where $\delta$ is the degradation factor.

When searching for solutions to soft CSP, it is possible to gain efficiency by not distinguishing values that could in any case not be part of a solution of sufficient quality. In $\alpha$ interchangeability, two values are interchangeable if they do not affect the quality of any solution with quality better than $\alpha$. We call $\alpha$ the threshold factor.

Both concepts can be combined, i.e. we can allow both degradation and limit search to solutions better than a certain threshold $\left({ }_{\alpha}^{\delta}\right.$ interchangeability).

By extending the previous definitions we can define thresholds and degradation version of full/neighbourhood substitutability/interchangeability.

As in the classical case, to find full soft interchangeability is computational expensive. However, there are local forms of neighbourhood interchangeability values which can be computed in polynomial time. Here are their definitions:

Definition 1. Consider two domain values $b$ and a for a variable $v$, the set of constraints $C$ and a semiring level $\delta$; we say that $b$ is ${ }^{\delta}$ Full Substitutable for a on $v$ $\left(b \in{ }^{\delta} F S_{v}(a)\right)$ if and only if for all assignments $\eta$,

$$
\bigotimes C \eta[v:=a] \times{ }_{S} \delta \leq_{S} \bigotimes C \eta[v:=b]
$$

We define values $a$ and $b$ to be ${ }^{\delta}$ Neighborhood Substitutable if the set of constraints $C$ is limited to the constraints including $V, C_{v}$. Further, we define $a$ and $b$ to be ${ }^{\delta}$ Full/Neighborhood Interchangeable if they are ${ }^{\delta}$ Full/Neighborhood Substitutable both ways.

Definition 2. Consider two domain values $b$ and $a$, for a variable $v$, the set of constraints $C$ and a semiring level $\alpha$; we say that $b$ is ${ }_{\alpha}$ Full substitutable for $a$ on $v$ $\left(b \in{ }_{\alpha} F S_{v}(a)\right)$ if and only if for all assignments $\eta$,

$$
\bigotimes C \eta[v:=a] \geq \alpha \Longrightarrow \bigotimes C \eta[v:=a] \leq_{S} \bigotimes C \eta[v:=b]
$$


Similarly to the Definition 1 , we define $a$ and $b$ to be ${ }_{\alpha}$ Neighborhood Substitutable if the set of constraints $C$ is limited to the constraints including $V, C_{v}$. Also, we define $a$ and $b$ to be ${ }_{\alpha}$ Full/Neighborhood Interchangeable if they are ${ }_{\alpha}$ Full/Neighborhood Substitutable both ways.

As Full Interchangeability needs a big computational effort we use Neighbourhood Interchangeability algorithms to compute interchangeable values, as they can compute in polynomial time and can well approximate Full Interchangeability ${ }^{2}$, see algorithms and proofs in [20] .

\section{Adaptation Model}

In previous work [1], we applied a more restricted interchangeability framework to case adaptation in resource allocation problems. In that approach it was possible to compute NI values which determine choices for a individual variable or a minimal NPI set which finds choices for a subset of variables of the CSP. In this work, we propose adaptation for the problems which are represented as soft constraint satisfaction problems where we use ${ }_{\alpha}^{\delta}$ interchangeability for updating solutions. In our model the adaptation is realised by interchanging values of one or a subset of variables of CSP by allowing a certain degradation of the solution, while there is no addition or deletion of the constraints of the CSP.

The adaptation model is illustrated here through an example application to a generic product configuration problem. The configuration of the product is defined as a CSP where:

- the components of the product are expressed as the variables of the CSP;

- the domains of the CSP are the sets of values that the components can be;

- the constraints among variables denote the dependency between the components in order that the product to be functional.

In our configuration example, we had used two representative forms of soft CSP : the weighted one to model the price and the fuzzy one to model the delivery time. So, for modelling the price of the product, the modelling of the CSP is the same as for the hard constraint case, only that we have cost for each value assignment of each component (unary constraint) and for each tuple combination over the constraints (binary constraint). By the use of the semiring operations for weighted CSP, $\left\langle\Re^{+}, \min ,+,+\infty, 0\right\rangle$ [11] for constraint combination we will have to add the costs in order to obtain the final price.

For delivery time we have used the fuzzy CSP model, where the semiring is defined as $<\Re^{+}, \min , \max ,+\infty, 0>^{3}$.

\subsection{The Architecture of the Adaptation Framework}

The architecture of the system is domain-independent; its domain knowledge comes from three external sources: a case library, domain knowledge represented as CSP and

\footnotetext{
${ }^{2}$ Also Neighbourhood Substitutability can approximate Full Substitutability

${ }^{3}$ Usually the fuzzy CSP is represented by the semiring $<[0,1], \max , \min , 0,1>$, but for modelling this example we had choosed a similar one which uses the opposite order
} 
user requirements, see Figure 3. Our sales manager system (shown in Figure 1), finds the appropriate product for a new buyer by retrieving cases and adapting their product configurations according to the new requests. The main contribution of this work relates to the adaptation step of the CBR process. Retrieval is done by using a simple comparison metric over the buyer profile relative to profiles contained in cases and picks out the closest previous cases.

As in Figure 3, the adaptation module receives the retrieved cases from the case base. The module interacts with the user for specific, personalised requirements and with the domain knowledge represented for making consistent adaptations.

The adaptation process proceeds as follows, see Figure 3:

- propose the retrieved cases to the user in order to make a choice;

- ask for the user for

- specific user requirements on the selected case;

- a certain price threshold or an allowed degradation of the price (specified as certain limits);

- a delivery time threshold or an allowed degradation of the delivery time (specified as certain limits);

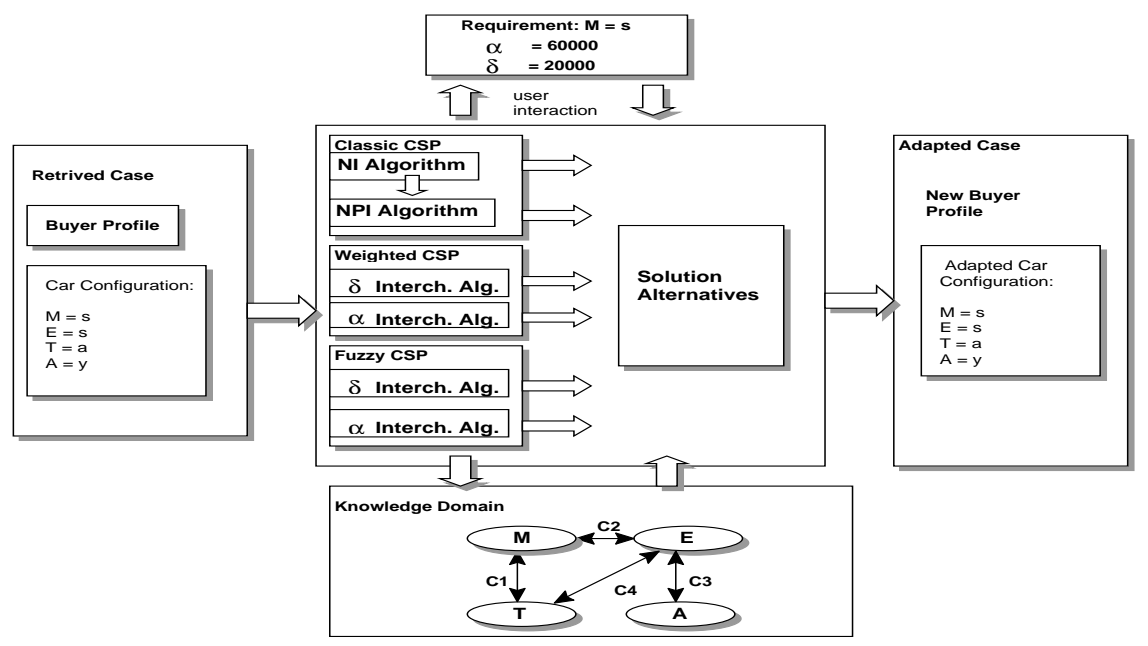

Fig. 3: Domain knowledge as Constraint Satisfaction Problems.

\subsection{Example - Degradation and Thresholds}

In the following we give an example of how degradation and threshold applies in order to update solutions when the domain knowledge is represented as a soft CSP.

The product catalog can represent the available choices through a soft CSPs, as show in Figure 2. By different representations of the semiring, the CSP represents different 
problem formulations. Thus, in order to model the cost of the product a weighted CSP might be most appropriate. For a weighted CSP, the semiring preference values model the cost of different components and their integration. The semiring operations and values are: $\left\langle\Re^{+}, \min ,+,+\infty, 0\right\rangle$. This allows one to model optimization problems where the goal is to minimize the total cost of the proposed solution. The cost of the solution is computed by summing up the costs of all constraints, intended as the cost of the chosen tuple for each constraint. Thus the goal is to find the n-tuples (where $n$ is the number of all the variables) which minimize the total sum of the costs of their subtuples (one for each constraint).
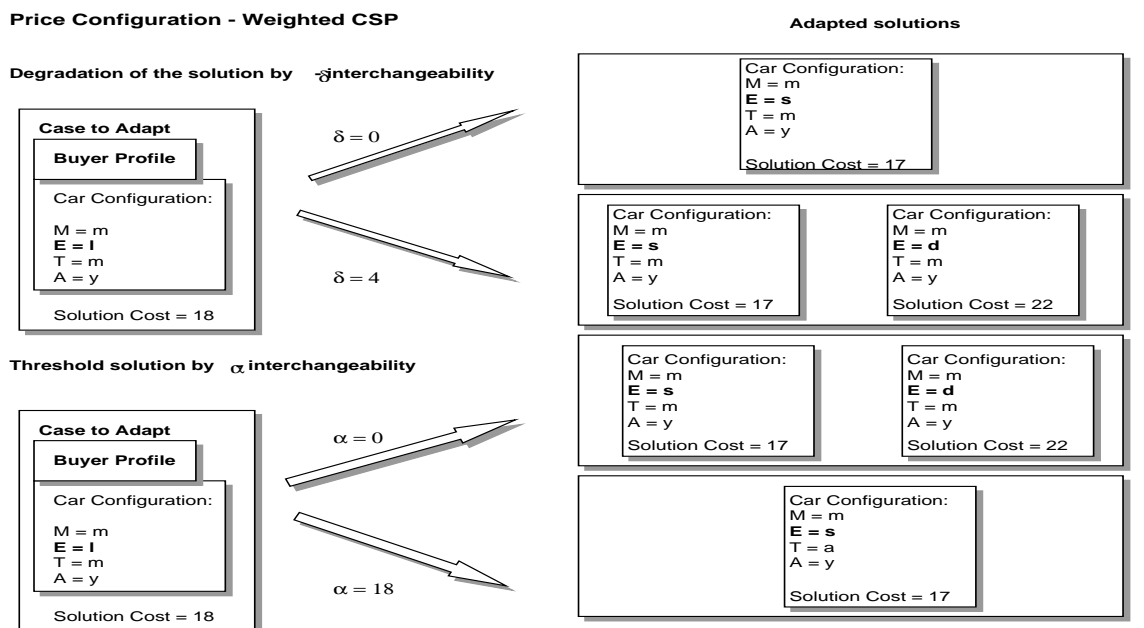

Fig. 4: Example of solution adaptation with NI interchangeability by a degradation $\delta$ or over a threshold $\alpha$ knowledge domain is represented as a Weighted CSPs.

In Figure 4, we present an example of solution update where the solution represents price modelling of the product modelled as a weighted CSP. The cost of the current solution is 18 and the user request is to update the value of the engine, here represented by variable $E$. The cost of the car in the current solution is 18 . By allowing a degradation of the solution with $\delta=0$ the solution updates to $\mathrm{E}=\mathrm{s}$ and a decrease in the cost to 17 . By allowing a degradation of $\delta=4$ solution can be adapted for variable $\mathrm{E}$ either to value $\mathrm{s}$ or $\mathrm{d}$ where the cost decreases to 17 or increases to 22 respectively. Among all the values of variable $\mathrm{E}$ we find that for degradation $\delta=0$, values s is ${ }^{0}$ substitutable to values 1 and $\mathrm{d}$, and 1 is ${ }^{0}$ substitutable to $\mathrm{d}$. For a degradation $\delta=1$, values s and 1 are ${ }^{1}$ interchangeable and ${ }^{1}$ substitutable to d. By allowing a degradation $\delta=4$, values s and 1 are ${ }^{4}$ interchangeable, values 1 and $d{ }^{4}$ interchangeable and $\mathrm{s}$ is ${ }^{4}$ substitutable to $\mathrm{d}$. For a degradation $\delta$ higher than 5 all the values for component $\mathrm{E}$ are ${ }^{\delta}$ interchangeable.

When searching for solutions to a soft CSP, it is possible to gain efficiency by not distinguishing values that could in any case not be part of a solution of sufficient quality. In $\alpha_{\alpha}$ interchangeability, two values are interchangeable if they do not affect the quality 
of any solution with quality better than $\alpha$, called the threshold factor. In our price modelling example, see Figure 4 we can see that for a threshold $\alpha=0$, the solution can be updated for variable $\mathrm{E}$ with all the other values of its domains as all its values are ${ }_{0}$ interchangeable; this is explained by the fact that since there are no solutions better than $\alpha=0$, by definition all the elements are interchangeable. For a certain threshold $\alpha$ $=18$, values 1 and $\mathrm{d}$ are ${ }_{18}$ interchangeable and s can substitute 1 and $\mathrm{d}$. And for higher $\alpha$, s can substitute 1 and $\mathrm{d}$ and $\mathrm{l}$ can substitute $\mathrm{d}$.
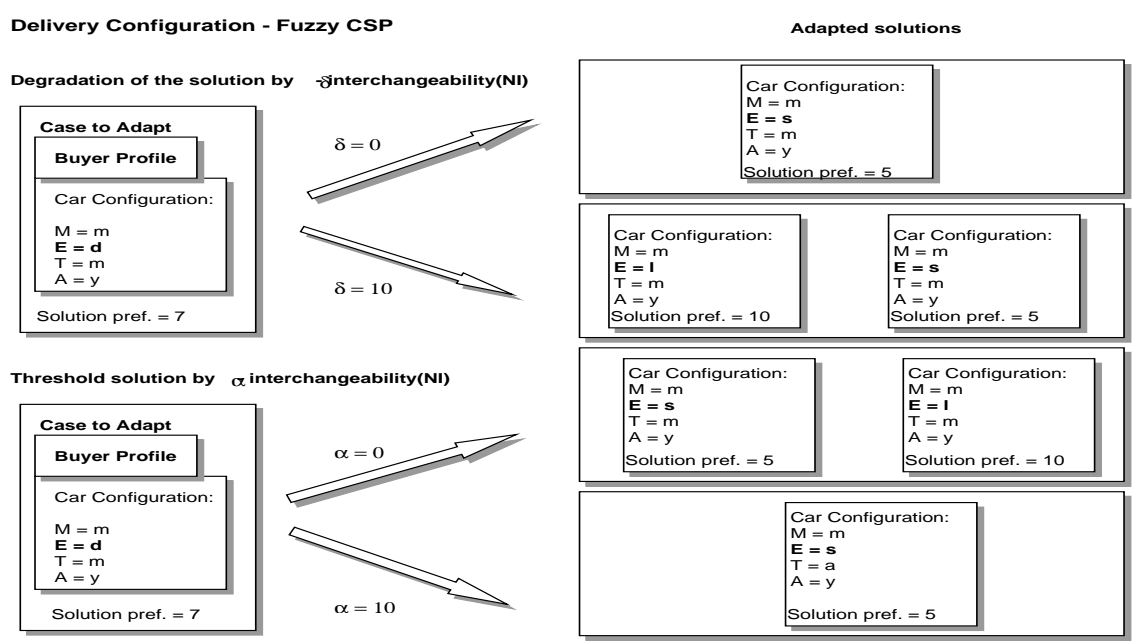

Fig. 5: Example of solution adaptation with NI interchangeability with a degradation $\delta$ or over a threshold $\alpha$ when the knowledge domain is represented as a Fuzzy CSPs.

For modelling the delivery time of the car we had used as optimisation criteria a fuzzy CSP. Fuzzy CSP associate a preference level with each tuple of values. Such level is usually between 0 and 1, where 1 represents the best value (that is the tuple is allowed) and 0 the worst, with the semiring $<[0,1]$, max, $\min , 0,1>$. In our example we had modelled the delivery time with the semiring $<\Re^{+}, \min , \max ,+\infty, 0>$ which is similar to the fuzzy one, but uses an opposite order. Let us called it opposite fuzzy. Delay in delivering time is determined by the time to obtain components and to reserve the resources for the assembly process. For the delivery time of the car, only the longest delay would matter. In Figure 5, the solution we have to adapt has a delivery time of 7 days but the user would prefer to change the engine type of the car. For a degradation of the solution $\delta=0$, we can adapt the solution only by value s for variable E. By allowing a higher degradation of the solution to $\delta=10$, there are two possible solution adaptation: when variable $\mathrm{E}$ takes value s the delivery time stays the same, while when it takes value 1 the delivery time increases to 10 days. For $\delta=0$, values s is ${ }^{0}$ substitutable for values 1 and $\mathrm{d}$ of variable $\mathrm{E}$, while for $\delta=7, \mathrm{~s}$ and $\mathrm{d}$ are becoming ${ }^{7}$ interchangeable and for $\delta=$ 10 all values of variable $\mathrm{E}$ are ${ }^{10}$ interchangeable. 
Computing adaptation by ${ }_{\alpha}$ interchangeability for this fuzzy example allows the adaptation of the solution, see Figure 5 to values s and 1 as well for variable $E$ as all the values are interchangeable for a threshold $\alpha$. For a threshold $\alpha=7$, values 1 and d stay interchangeable, but value s can only substitute 1 and d, while for higher values of $\alpha \mathrm{s}$ can substitute 1 and $d$ and only d can substitute 1 .

\section{Experimental Results}

In order to understand how frequently adaptation based on soft interchangeability would be successful, we have evaluated its occurrence on randomly generated problems. In the following, we give our first results about the occurrence of $(\delta / \alpha)$ interchangeability in soft CSPs.

We have done our experiments for fuzzy and weighted CSP representing the important class of Soft CSPs dealing with an idempotent and non-idempotent times operation respectively. The motivation for considering both classes comes from the fact that solving Soft CSP when the combination operation is not idempotent is extremely harder [17].

The occurrence of $(\delta / \alpha)$ interchangeability depends on the soft CSP structure. There are 4 standard parameters which characterize constraint problems:

- problem size, number of variables;

- domain sizes, number of values in the domain;

- problem density, the ratio of the number of constraints relatively to the minimum and maximum number of allowed constraints in the given problem;

- problem tightness, the ratio between the sum of the semiring values associated to all the tuples in all the constraints, and the value obtained by multiplying the $\mathbf{1}$ element of the semiring (that is the maximum) for the number of all possible tuple (that is the constraintnumber $\times$ domainsize).
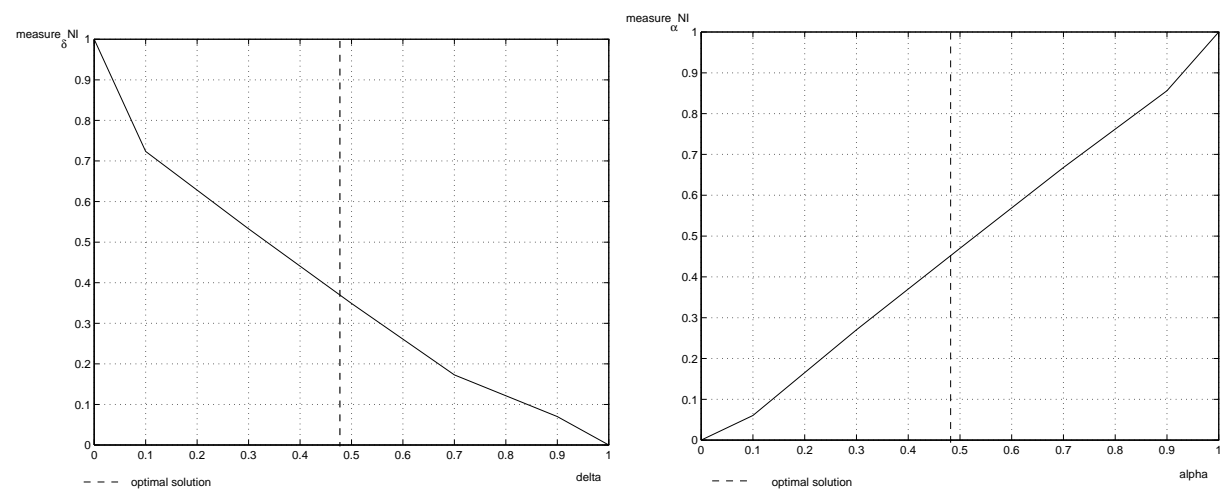

Figure 6: Occurrence of $\delta$ interchangeabil- Figure 7: Occurrence of $\alpha$ interchangeability for random generated Fuzzy CSPs. ity for random generated Fuzzy CSPs.

During our experiments on fuzzy and weighted CSPs, we had observed that the CSP density and the CSP size do not influence occurrence of interchangeable values. 
There is a weak dependency on the domain size, thus the number of interchangeabilities increases with the number of resources. There might be a strong dependency on the CSP tightness; it has to be proven in our further research. In Figure 6 and 7 we present our results for fuzzy CSP modelled with the semiring $<[0,1], \max , \min , 0,1>{ }^{4}$. In Figure 6 and 7, we see how interchangeability occurrence varies with $\delta$ and $\alpha$ respectively. The tests are done on sets of random generated problems with 10 variables, with varying density dens $-c s p \in\{0.1,0.2, \ldots, 1\}$ and the maximum domain size dom - size $=\left\{\frac{n}{10}, \frac{2 n}{10}, \ldots, \frac{9 n}{10}, n\right\}$, see model in [19].
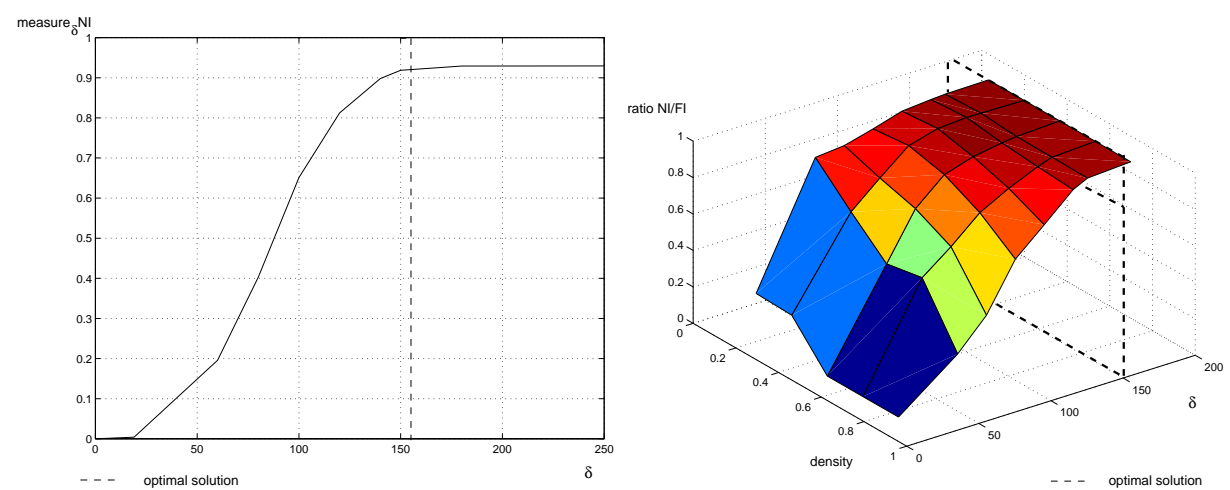

Figure 8: Occurrence of $\delta$ interchangeabil- Figure 9: Ratio between NI/FI $\delta$ interity for random generated Weighted CSPs. changeable values for random generated Weighted CSP.

The experiments show that for low semiring values there is a high occurrence of ${ }^{\delta} N I$ while it decreases till o for higher semiring values, Figure 6. The number of ${ }_{\alpha}$ interchangeable values is low for low semiring values and increases with the value of the semiring, Figure 7.

In all the graphs we highlight the position of the optimal solution. In fact, when dealing with crisp CSPs there does not exist any notion of optimality, but for soft CSPs each solution has an associated level. It is important to study NI occurrence around optimal solutions because we are often interested in discarding solutions of bad quality.

For $\alpha_{\alpha}$ interchangeability we did not find many interchangeabilities around optimal solution where the preference levels over the constraint tuples where uniformly generated. Although there is a high dependence of ${ }^{\delta} N I$ on the tightness of a soft CSP, it was not proved yet.

For ${ }_{\alpha}$ interchangeability we can see that around the optimal solution the measure of $\alpha$ interchangeable values goes from 0.1 to 0.6 .

Similar results hold for $\delta / \alpha$ interchangeability when dealing with weighted instead of fuzzy CSPs. In Figure 8, we represent ${ }^{\delta} N I$ occurrence in weighted CSPs. Notice

\footnotetext{
${ }^{4}$ Note that in our experiments we have used the semiring $<[0,1]$, $\max , \min , 0,1>$ but in the our example from Section 4 we have the opposite fuzzy defined with the semiring $<\Re^{+}, \min , \max ,+\infty, 0>$. However, these two models are equivalent and thus the results are relevant for both of them.
} 
that the shape of the function seems the opposite to that of Figure 6 only because the order in the fuzzy semiring (max-min) is opposite to the weighted semiring (min-sum). Similarly to fuzzy case, in weighted CSPs ${ }^{\delta}$ interchangeability decreases with $\delta$ (w.r.t. the semiring order), see Figure 8.

Computing full interchangeable values might be a quite costly operation as it may require computing all the solutions. It has been shown in [20] that full interchangeability $(\delta / \alpha F I)$ can be approximated by neighbourhood interchangeability $(\delta / \alpha N I)$.

Since $N I$ computes a subset of $F I$, it is important to investigate how many interchangeabilities we can find by computing locally neighbourhood interchangeabilities w.r.t. full interchangeabilities. In Figure 9 ratioNI / FI is represented the rate between Neighbourhood and Full Interchangeability for weighted CSPs. We can see that the ratio stays between 0.7 and 0.9 around optimal solution. In the fuzzy case, from our experiments we reached the conclusion that the ratioNI/FI stays always between 0.7 and 0.95 . Thus, $N I$ interchangeability can well approximate $F I$ interchangeability.

\section{Related Work}

In our previous work [1] we proposed a generic framework to realise case adaptation for the domain of problems which can be represented as crisp CSPs. Here we extend this framework for the domain of soft CSPs as well.

But there are some other approaches to seeing the adaptation problem as a constraint satisfaction problem. One of them was done by Purvis and $\mathrm{Pu}$ [21] in a casebased design problem solving domain based on the constraints over discrete CSPs. Their methodology formalises the case adaptation process in the sense of combining multiple cases in order to achieve the solution of the new problem, by applying repairbased CSP algorithm [22]. This method relies only on the knowledge accumulated in the case base where the method proposed by us in [1] considers also information offered by the domain knowledge formulated as CSP. The approaches are different as by method proposed by Purvis and $\mathrm{Pu}$ [21] the constraints between specific values of the variables are stored in the case, while adaptation method proposed by us in [1] considers in the case only the solution of the new problem, while the constraints between variables are held in an external module which contains the domain knowledge. We believe that our approach gives more flexibility to the adaptation module but we will study in the future how to combine the two methods for improving the adaptation process.

By extending the framework to the soft CSP domain and thus allowing preferences, the flexibility of the framework is further more improved.

Another approach of adaptation with constraints where the solution of a new problem is built by satisfying the new constraints and by transforming a memorised solution was done by Hua, Faltings and Smith [23] where they proposed a case-based reasoner for architectural design where constraints restrict numerical relationships among dimensions. CADRE introduced the concept of dimensional reduction: before attempting to adapt a case, it constructs an explicit representation of the degrees of freedom available for adaptation. However, CADRE defined this approach only for numeric constraints. The adaptation method based on dimensional reduction has been tested successfully in the IDIOM project [24]. 
In the domain of discrete variables, the adaptation space is less obvious. We proposed here a method based on interchangeability for domains of problems which can be expressed as soft CSPs which localise changes in discrete spaces and thus offers a reliable method for determining the closest solution.

\section{Conclusions and Future Work}

This paper presents methods for case adaptation for the domain of problems which can be represented soft constraint satisfaction problems. By combining with the adaptation methods for crisp CSPs in [1], we can obtain a powerful and generic case adaptation engine.

The adaptation methods for soft CSPs are generic and based on the soft interchangeability concept. We have proved the use of $(\delta / \alpha)$ substitutability/interchangeability in the adaptation process when the knowledge is represented as soft CSP. The two parameters $\alpha$ and $\delta$ allow us to express a wide range of adaptation alternatives. While the threshold $\alpha$ is used to eliminate distinctions that would not interest us anyway, the allowed degradation specifies how precisely we want to optimize the solution.

We presented here as well some preliminary results which measure the occurrence of $(\delta / \alpha)$ interchangeability thus number of adaptation alternatives. There is a high occurrence of ${ }_{\alpha} N I$ interchangeability around optimal solution and one can use successfully $\delta$ interchangeability for low degradation of the solution, but further study has to be done on that. Moreover, we also show that NI interchangeability, a computable interchangeability method, can well approximate $F I$ interchangeability.

Interesting future directions include:

- algorithms for computing neighbourhood partial interchangeability for soft CSPs;

- further experimental tests to measure dependence of $(\delta / \alpha)$ interchangeability on soft CSP tightness.

- Classifying case bases of solutions by how re-usable ("adaptable") their solutions are according to allowed $\delta$ degradation and/or solution quality relatively to a threshold $\alpha$.

\section{References}

1. Nicoleta Neagu and Boi Faltings. Exploiting Interchangeabilities for Case Adaptation. In Proc. of the 4th ICCBR01, 2001.

2. Stefano Bistarelli, Boi Faltings, and Nicoleta Neagu. A Definition of Interchangeability for Soft CSPs. In ERCIM Workshop(to appear)., Cork, Ireland, 2002.

3. Van Hentenryck. Generality versus Specificity: An Experience with AI and OR Techniques. In AAAI-88: Proceedings National Conference on Artificial Intelligence, pages 660-664, 1988.

4. R Dechter Y. El Fattah. Diagnosing tree-decomposable circuits. In Proc. of the 14 th IJCAI, pg. 1742-1748, 1995.

5. H. Krautz and B. Selman. Planning as Satisfiability. In Proc. of the 10 th Ecai, pages 359-363, Vienna, Austria, 1992. 
6. M. Wallace. Applying constraints for scheduling. In Constraint Programming, volume 131 of NATO ASI Series Advanced Science Institute Series. Springer Verlag, 1994.

7. A. Mackworth. Constraint-based Design of Embeded Intelligent Systems. In Constraints 2(1), pages 83-86, Vienna, Austria, 1992.

8. R. Weigel and B. Faltings. Interchangeability for Case Adaptation in Configuration Problems. In Proceedings of the AAAI98 Spring Symposium on Multimodal Reasoning, Stanford, CA, TR SS-98-04., 1998.

9. T. Schiex. Probabilistic constraint satisfaction problems, or "how to handle soft constraints?" . In Proc. 8th Conf. of Uncertanty in AI, 269-275, 1992.

10. Stefano Bistarelli, Ugo Montanari, and Francesca Rossi. Semiring-based Constraint Solving and Optimization. Journal of ACM., 44, n.2:201-236, 1997.

11. Stefano Bistarelli, Boi Faltings, and Nicoleta Neagu. Interchangeability in Soft CSPs. In Proc. of the 8th CP-2002., Ithaca, NY, USA, 2002.

12. E.C. Freuder and R.J. Wallace. Partial constraint satisfaction. AI Journal, 58, 1992.

13. D. Dubois, H. Fargier, and H. Prade. The calculus of fuzzy restrictions as a basis for flexible constraint satisfaction. In Proc. IEEE International Conference on Fuzzy Systems, pages 1131-1136. IEEE, 1993.

14. Zs. Ruttkay. Fuzzy constraint satisfaction. In Proc. 3rd IEEE International Conference on Fuzzy Systems, pages 1263-1268, 1994.

15. H. Fargier and J. Lang. Uncertainty in constraint satisfaction problems: a probabilistic approach. In Proc. European Conference on Symbolic and Qualitative Approaches to Reasoning and Uncertainty (ECSQARU), volume 747 of LNCS, pages 97-104. Springer-Verlag, 1993.

16. T. Schiex, H. Fargier, and G. Verfaille. Valued Constraint Satisfaction Problems: Hard and Easy Problems. In Proc. IJCAI95, pages 631-637, San Francisco, CA, USA, 1995. Morgan Kaufmann.

17. S. Bistarelli, U. Montanari, and F. Rossi. Semiring-based Constraint Solving and Optimization. Journal of the ACM, 44(2):201-236, Mar 1997.

18. S. Bistarelli, U. Montanari, and F. Rossi. Semiring-based Constraint Logic Programming: Syntax and Semantics. ACM Transactions on Programming Languages and System (TOPLAS), 23:1-29, jan 2001.

19. Berthe Choueiry Boi Faltings, Rainer Weigel. Abstraction by Interchangeability in Resource Allocation. In Proc. of the 14 th IJCAI-95, pages 1694-1701, Montreal, Canada, 1995.

20. S. Bistarelli, B. Faltings, and N. Neagu. A definition of interchangeability for soft csps. In Proc. of the Joint Workshop of the ERCIM Working Group on Constraints and the CologNet area on Constraint and Logic Programming on Constraint Solving and Constraint Logic Programming - Selected Papers, LNAI. Springer-Verlag, 2002.

21. L. Purvis and P. Pu. Adaptation using Constraint Satisfaction Techniques. In Proc. of the 1st International Conference in CBR, pages 88-97, 1995.

22. A. Philips S. Minton, M. Johnson and P. Laird. Minimizing Conflicts: A Heuristic Repair Method for Constraint Satisfaction and Scheduling Problems. In Artificial Intelligence 58, pages 88-97, 1995.

23. B. Faltings K. Hua and I. Smith. CADRE: case-based geometric design. In Artificial Intelligence in Engineering 10, pages 171-183, 1996.

24. C. Lottaz I. Smith and B.Faltings. Spatial composition using cases: IDIOM. In Proc. of the 1st International Conference in CBR, pages 88-97, 1995. 